\title{
Managing the Journey of Patients under Chemotherapy in a Pandemic Era: A Nursing Perspective
}

\author{
Raffaella Gualandi Anna De Benedictis Maria Grazia De Marinis Daniela Tartaglini \\ Department of Nursing, Università Campus Bio-Medico di Roma, Rome, Italy
}

\section{Key Messages}

- This epidemic offers numerous opportunities for improving the care of patients undergoing chemotherapy and cancer patients in general. Understanding when the patient needs to go to the hospital and mitigating the risk of a lack of continuity of care through technology is one of the biggest challenges. Nursing competence can offer a valid contribution in assessing the patient's risk of contagion and the remote monitoring of the patient's needs.

\section{Keywords}

Chemotherapy · Patient journey · Pandemic · COVID-19 • Nursing

\section{Abstract}

Background: During the COVID-19 pandemic, cancer patients' care needs to be reconsidered by integrating the patient's clinical pathway with the hospital patient journey and the family context in a safe and patient-centered way. So far, no systematic reports are available regarding the impact of the COVID-19 pandemic on cancer care. This work gives a first overview of patients' care needs undergoing chemotherapy treatment from a nursing perspective.

(c) 2021 S. Karger AG, Basel

\section{Introduction}

The COVID-19 pandemic is straining the care modality of patients receiving chemotherapy treatments. The greater vulnerability of cancer patients, the alteration of treatment pathways, and the interruption of clinical trials are just some of the effects that the burden of COVID-19 has had on cancer patients' care $[1,2]$. Health professionals are faced with an unprecedented challenge of treating cancer patients while also ensuring their safety and aspirations for the best treatment possible. Therefore, during the COVID-19 pandemic, the care of cancer patisents needs to be reconsidered to integrate the patient's clinical pathway with the hospital patient journey and the family context in a safe and patient-centered way [3].

Although limited studies and research exist on the experience of this particular patient population, it is reasonable to think that in treated cancer patients, there may be significant needs that emerged during this pandemic as a consequence of restrictive regulations and changes in hospital pathways [1]. If, on the one hand, numerous recommendations have been made to safeguard the patient's health from the risk of COVID-19 contagion, on the other, it is not yet clear what are the care needs of patients in a changed healthcare context, if new ones have emerged, and how they are treated. karger@karger.com www.karger.com/che

(c) 2021 S. Karger AG, Basel

Karger"
Anna De Benedictis

Department of Nursing, Università Campus Bio-Medico di Roma Via Álvaro del Portillo, 21 IT-00128 Rome (Italy)

a.debenedictis@unicampus.it 
So far, no systematic reports are available regarding the impact of the COVID-19 pandemic on cancer care from a nursing perspective. The purpose of this paper is to report the current knowledge on the needs, experiences, and responses to the treatment of patients treated with chemotherapy in a context at a high risk of contagion. Results are showed to stimulate further research on how the health system, and the nursing sector in particular, can meet the needs of patients undergoing chemotherapy treatment in a pandemic era.

\section{Methods}

A narrative review has guided this work due to the very recent and pressing health crisis and the urgency to stimulate adequate responses. This method helps to present a broad perspective of a topic by pulling currently available pieces of literature together [4]. The first author searched PubMed and Scopus for papers published until October 2020 to identify all relevant articles reporting patients' needs undergoing chemotherapy treatment related to the pandemic situation. Additional publications were searched in the references cited in the initial papers. A comprehensive search was performed using the following search terms: (chemotherapy OR anticancer therapy) AND (SARS-CoV-2 OR 2019-nCoV OR COVID-19 OR Coronavirus disease 2019 OR Severe acute respiratory syndrome coronavirus) AND (clinical pathway OR patient journey). Studies included in this review had to meet the following criteria: (i) articles on adult patients undergoing chemotherapy treatment during a pandemic; (ii) reviews examining patient needs or patient nursing care; and (iii) articles in English language and published in a peer-reviewed journal with abstract available. Considering the paucity of scientific evidence, commentaries, perspectives, case reports, and editorial letters were included in the search. The following studies were excluded: (i) articles on clinical characteristics in patients undergoing chemotherapy and with COVID-19; (ii) articles on treatment options for cancer patients with COVID-19; and (iii) articles on COVID-19 therapies and anticancer drugs.

After removing duplicates, titles and abstracts were then screened to exclude citations that did not meet the inclusion criteria. After the initial screening, all remaining full-text articles were analyzed to perform a narrative synthesis of results.

\section{Rethinking the Patient Journey}

In the last months, the most prominent challenge the healthcare professionals are faced with is to protect patients with cancer from infection while ensuring the care they need. Two main variables are to be considered to achieve this goal. On the one side, cancer patients have a 2 -fold increased risk of COVID-19 infection when compared with the general population [5], and the hospital is one of the places where the risk of contagion is high. On the other, patients undergoing chemotherapy treatment have to make numerous admissions to the hospital.

Health professionals' efforts immediately focused on identifying patients at major risk of contagion to minimize hospital access. Therefore, the need for accurate telephone triage before each access to the hospital was immediately evident $[1,3]$. Moreover, a risk as- sessment score was developed for patients with cancer by combining clinical and laboratory variables to weigh the risk of COVID-19 [6].

Telemedicine has been widely implemented to reduce the risk of contagion among patients [7]. A report published in May 2020 by The Lancet - Oncology describes the very first experiences adopted to manage patients with cancer in Wuhan [8]. A fast implementation of telemedicine services allowed patients receiving anticancer treatments to use internet or telephone services as the first choice to contact their doctors rather than going directly to the hospital. However, while telemedicine can solve patient monitoring, it does not solve the problem of administering chemotherapy. Some authors suggested a home-drawn blood service and home infusion of chemotherapy, if medically and logistically feasible [1]. Other authors reported using a courier service or a drive-through medicine dispensing facility for the administration of oral chemotherapy [9]. A study of 435 cancer patients reported on how patient care was modified, which resulted in a canceled or adapted treatment. This occurred in $44 \%$ of the patients receiving systemic treatment. Of them, $48 \%$ had an adaptation of oral chemotherapy and $46 \%$ had an interruption or cuts in immunotherapy [10].

No data are available on how patients have adapted to the indication of reducing hospital access, although some authors report how new patients may be worried about whether their treatments will be delayed and what the implications might be on their outcome [11]. However, a study carried out during the SARS epidemic reported how two-thirds of 79 patients with non-small cell lung cancer were reluctant to accessing the hospital for concerns about infecting with SARS and 3 patients terminated any further chemotherapy [12].

Patients assigned to clinical trials also changed their care pathway by simplifying trial-related procedures and reducing the operational burden [13]. Minimizing the number of protocol-required procedures (including blood tests and hospital visits), performing telemedicine consultations, allowing for shipping oral investigational medications, and modifying treatment schedules for intravenous drugs were some of the suggested interventions [2, 14]. No studies were found on the impact of delay or change of therapeutic path on patients enrolled in clinical trials in terms of patient expectations, anxiety about the likelihood of protocol success, and home management of symptoms.

\section{Maintaining the Patient-Health Professional Relationship}

A decreased number of hospital visits, limited to the days of drug infusion, could significantly increase patients' perception of inefficacious or incomplete communications with the clinicians. Moreover, a nonsystematic clinical assessment could lead to missing important information about patients' symptoms and treatment side effects [15]. Direct-to-consumer (or on-demand) telemedicine can allow physicians and patients to communicate $24 \mathrm{~h} / 7$ days. Some practical measures have been reported to ensure continuity in the relationship between the patient and healthcare professionals, such as archiving contact details of all patients (including e-mail address and mobile telephone number), rapid updates with the latest advice via e-mail and/or text, and warnings to patients on the actual availability of health personnel on the channels depending upon staff capacity [11]. However, no studies demonstrate the effectiveness of using these technologies in maintaining a good relationship between the patient and the healthcare professional. The use of electronic patient-reported outcomes can help professionals in the screening of patients who need an additional telephone interview or direct medical intervention [15]. 
Fig. 1. Ensuring the cancer patient care undergoing chemotherapy during a pandemic.

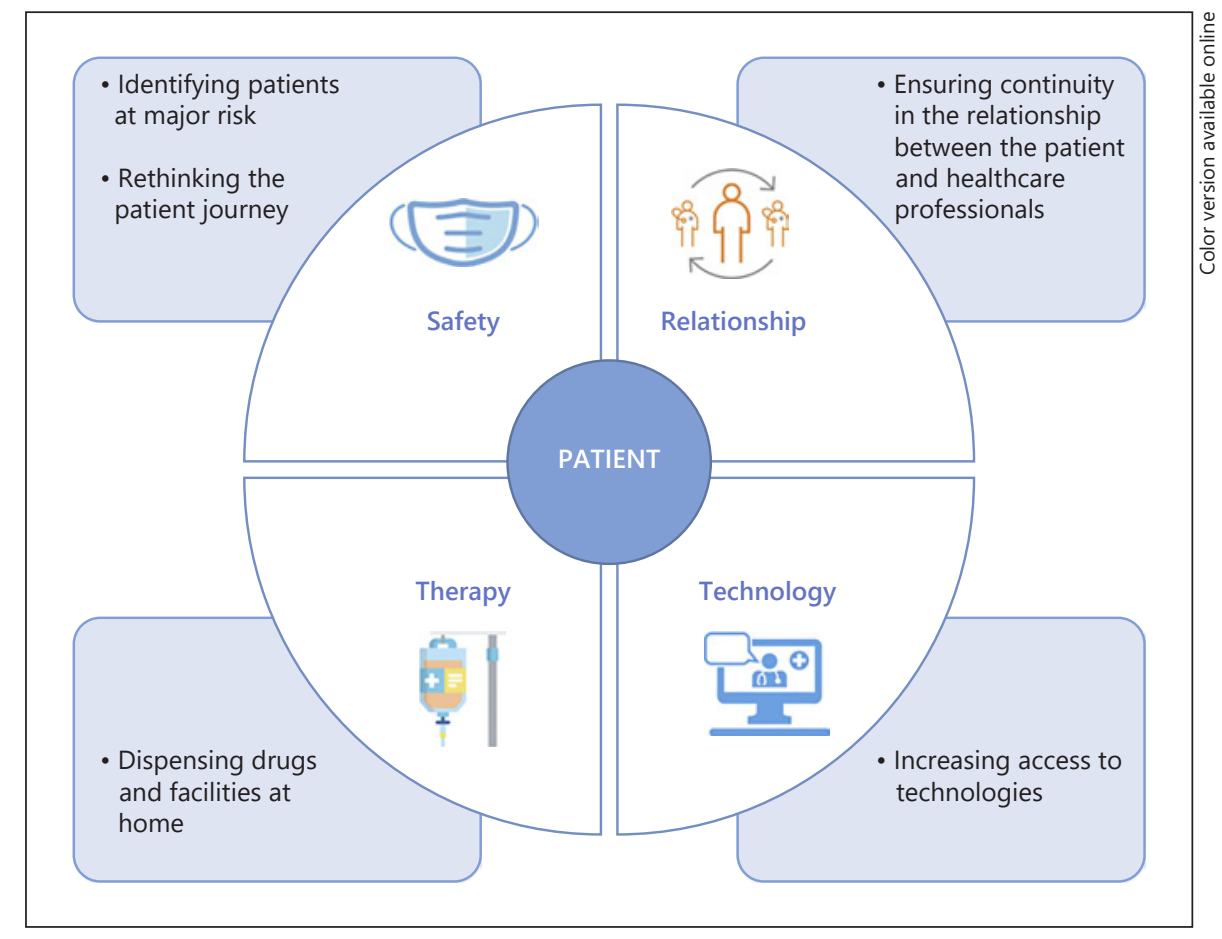

\section{Treating the Risk of Patient Isolation}

As a result of imposed quarantine and social distancing, friends and relatives are discouraged from visiting inpatients or accompanying patients to clinic appointments. Consequently, family members may be limited in providing patient support and advocacy. An additional life-threatening condition can lead the patient to isolating from family environment. Consequently, anxiety and depression, already common in patients undergoing chemotherapy, can be exacerbated by the situation of isolation [11].

Older cancer patients are part of a population more at risk of isolation, resulting in physical and psychological deterioration. They may experience feelings of estrangement and neglect due to limited access to news or information, friends, and family. This issue is further exacerbated due to the difficulty of accessing digital technology. A decline in communication and comprehension can be a consequence of wearing masks and face shields, particularly for hard-of-hearing patients who rely on lip-reading and nonverbal cues [16]. Authors recommend using psychosocial staff to assess distress and to address patients' and families' ongoing needs during this pandemic [11]. However, no other studies address this issue and mean to minimize the consequences of patient isolation during a pandemic. Figure 1 summarizes the key factors to ensure the cancer patient care undergoing chemotherapy during a pandemic.

\section{Conclusions}

In times of health emergency, healthcare professionals are the first to deal with patients' pressing needs. As the numbers of patients with COVID-19 are increasing, new solutions to emerging problems need to be implemented even without scientific evidence. The experience gained on field is now essential to define guidelines to adopt when acting in an epidemic scenario. In this narrative review, some of the current challenges associated with the caring for cancer patients undergoing chemotherapy during a pandemic are provided. Since the emergence of the novel coronavirus SARS-CoV-2 (COVID-19), oncology patients' care has changed dramatically $[8,17]$. In particular, cancer patients undergoing or candidates for chemotherapy are regarded as a highly vulnerable group.

Recent literature is mainly addressing the virology, diagnosis, and treatment of cancer patients affected by COVID-19. However, limited studies are available on what the patient has experienced during this period and what needs have remained unmet. While the primary commitment has been to save as many lives as possible, it is now necessary to find long-term strategies to help patients to better adapt to the new conditions of care. This epidemic reveals numerous opportunities for improving the care of patients undergoing chemotherapy. Understanding when the patient needs to go to the hospital and mitigating the risk of a lack of continuity of care through the use of technology are the biggest challenges [18]. Nursing competence can offer a valid contribution in assessing the patient's risk of contagion and providing a remote monitoring of the patient's needs. Patient-reported outcomes, 
and patient experience data in particular, should be essential tools for understanding the patient's real situation and for planning specific integrated interventions (i.e., home delivery of drugs and aids, telephone calls from volunteers, and patient and caregiver education in managing the risk of infection) [19].

Literature regarding the long-term impact of pandemics on patients undergoing chemotherapy will have to arise from the next 2-year surveys. However, by immediately capturing possible variables that can affect patients survival and quality of life (e.g., postponement of treatment, isolation, and lack of a caregiver who knows how to identify the patient's care needs), it will be possible to understand how to improve the care of patients undergoing chemotherapy during a pandemic.

Research is more necessary than ever to prepare the health professionals of the future to mitigate the negative impact of a pandemic on cancer patient outcomes. A patient-centered approach will be a key to success.

\section{Conflict of Interest Statement}

The authors have no conflicts of interest to declare.

\section{Funding Sources}

The authors received no specific funding for this work.

\section{Author Contributions}

Gualandi Raffaella conceived and designed the study; collected the data and performed the analysis; and wrote the paper. De Benedictis Anna revised the work critically for important intellectual content; discussed the results; and contributed to the final manuscript. De Marinis Maria Grazia and Tartaglini Daniela revised the work critically for important intellectual content.

\section{References}

1 Al-Shamsi HO, Alhazzani W, Alhuraiji A, Coomes EA, Chemaly RF, Almuhanna M, et al. A practical approach to the management of cancer patients during the novel coronavirus disease 2019 (COVID-19) pandemic: an International Collaborative Group. Oncologist. 2020 Jun;25(6):e936-45.

2 Richards M, Anderson M, Carter P, Ebert BL, Mossialos E. The impact of the COVID-19 pandemic on cancer care. Nat Cancer. 2020:1-3.

3 Elkin E, Viele C, Schumacher K, Boberg M, Cunningham M, Liu L, et al. A COVID-19 screening tool for oncology telephone triage. Support Care Cancer. 2020:1-6.

4 Green BN, Johnson CD, Adams A. Writing narrative literature reviews for peer-reviewed journals: secrets of the trade. J Chiropr Med. 2006;5(3):101-17.

$5 \mathrm{Yu}$ J, Ouyang W, Chua MLK, Xie C. SARSCoV-2 transmission in cancer patients of a tertiary hospital in Wuhan. JAMA Oncol. 2020 Jul 1;6(7):1108-10.

6 Indini A, Rijavec E, Ghidini M, Cattaneo M, Grossi F. Developing a risk assessment score for patients with cancer during the coronavirus disease 2019 pandemic. Eur J Cancer. 2020;135:47-50.

7 Monaghesh E, Hajizadeh A. The role of telehealth during COVID-19 outbreak: a systematic review based on current evidence. BMC Public Health. 2020;20(1):1193.
8 Mei H, Dong X, Wang Y, Tang L, Hu Y. Managing patients with cancer during the $\mathrm{CO}$ VID-19 pandemic: frontline experience from Wuhan. Lancet Oncol. 2020 May;21(5):6346.

9 Willan J, King AJ, Hayes S, Collins GP, Peniket A. Care of haematology patients in a COVID-19 epidemic. Br J Haematol. 2020 Apr;189(2):241-3.

10 Helissey C, Cessot A, Boudin L, Romeo E, Prieux C, Ghebriou D, et al. Evaluation of medical practices in oncology in the context of the COVID-19 pandemic in France: physicians' point of view: the PRATICOVID study. Cancer Med. 2020 Dec;9(23):8875-83.

11 Paterson C, Gobel B, Gosselin T, Haylock PJ, Papadopoulou C, Slusser K, et al. Oncology nursing during a pandemic: critical reflections in the context of COVID-19. Semin Oncol Nurs. 2020;36(3):151028.

12 Chen YM, Perng RP, Chu H, Tsai CM, Whang-Peng J. Impact of severe acute respiratory syndrome on the status of lung cancer chemotherapy patients and a correlation of the signs and symptoms. Lung Cancer. 2004; 45(1):39-43.

13 Moujaess E, Kourie HR, Ghosn M. Cancer patients and research during COVID-19 pandemic: a systematic review of current evidence. Crit Rev Oncol Hematol. 2020;150: 102972.
14 Fontana E, Arkenau HT. Oncology clinical trials during the COVID-19 outbreak: lessons learnt during the crisis and future opportunities. Cancer Treat Rev. 2020;88:102047.

15 Marandino L, Necchi A, Aglietta M, Di Maio M. COVID-19 emergency and the need to speed up the adoption of electronic patientreported outcomes in cancer clinical practice. JCO Oncology Practice. 2020;16(6):295-8.

16 Battisti NML, Mislang AR, Cooper L, O’Donovan A, Audisio RA, Cheung KL, et al. Adapting care for older cancer patients during the COVID-19 pandemic: recommendations from the International Society of Geriatric Oncology (SIOG) COVID-19 working group. J Geriatr Oncol. 2020;11(8):1190-8.

17 Hen N, Zhou M, Dong X, Qu J, Gong F, Han $\mathrm{Y}$, et al. Epidemiological and clinical characteristics of 99 cases of 2019 novel coronavirus pneumonia in Wuhan, China: a descriptive study. Lancet. 2020;395(10223):507-13.

18 Hollander JE, Carr BG. Virtually perfect? Telemedicine for Covid-19. N Engl J Med. 2020 Apr 30;382(18):1679-81.

19 Millstein BH, Kindt S. Reimagining the patient experience during the COVID-19 pandemic. 2020. 